\title{
Application of Adaptive Quadrature to Axi-symmetric Vortex Sheet Motion
}

\author{
Qing Nie \\ Institute for Mathematics and Its Applications \\ 514 Vincent Hall \\ 206 Church Street S.E. \\ Minneapolis, MN 55455-0436 \\ nie@ima.umn.edu \\ Greg Baker \\ Department of Mathematics \\ Ohio State University \\ Columbus, Ohio 43210-1174 \\ baker@math.ohio-state.edu
}

May 9, 1997

Subject Classification: 65R20,76B99

Keywords: Axisymmetric Vortex Sheets, Adaptive Integration. 
Running Head: Adaptive Quadrature for Vortex Sheets

Send proofs to:

Prof. Greg Baker

Department of Mathematics

Ohio State University

231 W. 18th Ave

Columbus, $\mathrm{OH} 43210$ 


\begin{abstract}
Studies of the formation of fine structures on free-surfaces in liquids, such as curvature singularities or interface pinching, demand that the motion of the interface must be computed very accurately. Boundary integral techniques are a popular choice in such studies because they reduce the dimension of the problem by one. On the other hand, the boundary integrals are singular, and their accurate evaluation can prove quite challenging. In two dimensional motion, the interface is a just a curve. When this curve is closed or periodic, the singularity in the integrand may be removed and the trapeziodal rule may be applied with spectral accuracy. Unfortunately, the nature of the singularity in the integrand for three dimenional motion is much more difficult to treat. In this paper, we present an accurate adaptive quadrature to compute the motion of a vortex sheet in axi-symmetric flow. The technique is based on a vector-potential formulation which offers some computational advantages over other methods based on the Biot-Savart Integral. Direct numerical computations show that our technique is much more accurate and efficient than existing techniques.
\end{abstract}




\section{Introduction}

Boundary integral techniques provide a popular approach to studying free-surface motion in liquids, covering a wide range of phenomena. Some examples are the propagation of waves in inviscid liquids [1] and the motion of drops in very viscous

liquids [2]. One of the major features of boundary integral technique is that they provide an evolution equation for the motion of the interface explicitly. Since there is no need to determine the flow field away from the interface, the dimension of the problem is reduced by one. On the other hand, the boundary integrals are principal-value integrals and their accurate evaluation can be very challenging. This is particularly true in studies of the formation of fine structures on the interface, such as curvature singularities or interface pinching.

In two dimensional motion there have been several successful studies of interfacial motion. In particular, spectrally accurate methods are available when the interface is closed (as in rising bubbles [3]) or is periodic in open geometry (as in water waves [1] or Rayleigh-Taylor instability [4]). First, the pole singularity in the principalvalue integral may be removed by the subtraction of a simple integral, and then the trapeziodal rule may be applied with spectral accuracy to the resulting periodic integral [5], [6]. The high order of accuracy proves very desirable in studies of fine features, such as the formation of a curvature singularity in vortex sheet motion [7] or during the Rayleigh-Taylor instability [8].

The situation is very different for the computation of axi-symmetric flows. The principal-value integrals involve complete elliptic integrals which contain logarithmic singularities. The singularity in the integrand can be weakened, but cannot be removed completely as in the two dimensional case. Further, the integrand shows strong variations, especially near the poles [9], which makes the design of an accurate quadrature difficult. For example, a vortex ring method [10] with a correction term to treat the principal-value also finds diffculties at the pole: the order of the method is reduced to $O(h)$ near the poles where $h$ is the spacing between the rings. An improvement to this method has been found recently [11] by an ingenious ana- 
lytic approximation to the integrand near the poles. The improved method is still only $O\left(h^{3} \log h\right)$. A different approach [9], [12] is to inset more quadrature points through interpolation when performing the integration for points near the poles, but numerical instabilities appear in the vortex sheet near the poles after a short time. This behavior illustrates a useful principle in designing methods for vortex sheet motion: Not only must the numerical errors from the boundary integrals be small, they should also be relatively smooth to avoid the onset of instabilities.

There have been attempts [13], [14] to study the formation of curvature singularities in axi-symmetric vortex sheets. The numerical calculations were based on the vortex ring method [10], and while evidence for the existence of the singularity was found, not enough resolution was possible to identify the nature of the singularity. In our experience, it is necessary to compute the boundary integral to an accuracy of about $10^{-20}$ or better to determine the precise form of the singularity. This requirement is probably too severe even for methods of moderate order. In this paper, we present efficient and accurate integration for axi-symmetric vortex sheet computations based on high order adaptive Gauss-Kronrod or Clenshaw-Curtis quadratures.

We use a dipole representation for a vortex sheet, rather than the conventional vorticity distribution on the sheet, because it is easier to weaken the singularity in the principal-value integrals by the subtraction of a suitable analytic expression. Then we apply adaptive numerical integration methods to the integrals of the dipole distribution through two different approaches. In the first approach, we use GaussKronrod quadrature directly on the integrals in the standard adaptive way. In the second approach, we first approximate the two complete elliptic integrals in the integrands by the sum of two parts, a regular part and a logarithmic part; then we apply Gauss-Kronrod quadrature adaptively to the regular part and the modified Clenshaw-Curtis quadrature adaptively to the logarithmic part.

We test these methods and compare them to the vortex ring method [10] on a standard test case, the flow around a spherical vortex sheet held fixed in time. By comparing the calculations of the velocity on the sheet, we find that even the non-adpative Gauss-Kronrod outperforms the vortex ring method. The adaptive 
Gauss-Kronrod and Clenshaw-Curtis quadratures are comparable and much better than the non-adaptive methods. The adaptive Clenshaw-Curtis method is the most efficient by far.

The organization of the paper is as follows. In Section 2, we derive the equations of motion for a full three dimensional vortex sheet and its axi-symmetric version. We give both formulations, the one based on the Biot-Savart law, and the other based on the dipole distribution. We describe the numerical techniques in Section 3, and discuss and compare the performance of the different methods in Section 4. Finally, we present the results of the calculation of the motion of an axi-symmetric sheet using the adaptive Clenshaw-Curtis quadrature. We find no evidence of numerical instabilities.

\section{Formulations}

In this section, we give the boundary integral equations for a three dimensional vortex sheet, then we obtain the special form for an axi-symmetric sheet. We follow the same notation as in [15], but we derive the equations from a different point of view based on representing the vortex sheet by a dipole distribution.

Let $S$ denote a closed vortex sheet, which divides the fluid region into two parts: an outside region $D_{+}$and an inside region $D_{-}$. The velocity and pressure fields are $\left(u_{ \pm}, p_{ \pm}\right)$, respectively. The Euler equations of motion are:

$$
\begin{gathered}
\frac{\partial \mathbf{u}_{ \pm}}{\partial t}+\mathbf{u}_{ \pm} \cdot \nabla \mathbf{u}_{ \pm}+\nabla p_{ \pm}=0 \quad \text { in } D_{ \pm} \\
\nabla \cdot \mathbf{u}_{ \pm}=0 \quad \text { in } D_{ \pm} \\
\nabla \times \mathbf{u}_{ \pm}=0 \quad \text { in } D_{ \pm} / S .
\end{gathered}
$$

Kinematic and dynamic considerations require the following conditions on the vortex sheet:

$$
\mathbf{n} \cdot \mathbf{u}_{+}=\mathbf{n} \cdot \mathbf{u}_{-} \quad \text { and } \quad p_{+}=p_{-} \quad \text { on } S
$$

where $\mathbf{n}$ is the normal to $S$ pointing into $D_{+}$. It is the jump in tangential components of the velocity that characterizes the vortex sheet. 
We introduce two surface coordinates $\alpha$ and $\beta$ and express the location of the interface as $\mathbf{X}(\alpha, \beta, t)$. These coordinates will be defined by the requirement that the motion of the sheet is "Lagrangian." By that we mean a "particle" on $S$ labelled by $\alpha$ and $\beta$ moves with the velocity

$$
\frac{\partial \mathbf{X}}{\partial t}=\mathbf{u} \equiv \frac{1}{2}\left(\mathbf{u}_{+}+\mathbf{u}_{-}\right) \quad \text { on } S
$$

Note that the normal component of the velocity of the sheet matches that of the two liquids as required by (4).

Due to $(2,3)$, there exist potential functions $\phi_{ \pm}$such that

$$
\mathbf{u}_{ \pm}=\nabla \phi_{ \pm} \quad \text { in } D_{ \pm} / S .
$$

We introduce a dipole distribution $\mu(\alpha, \beta, t)$ on the sheet. It generates a potential function

$$
w(\mathbf{x})=-\frac{1}{4 \pi} \int_{S} \mu\left(\mathbf{x}^{\prime}\right) \mathbf{n}\left(\mathbf{x}^{\prime}\right) \cdot \nabla_{\mathbf{x}^{\prime}}\left(\frac{1}{\left|\mathbf{x}-\mathbf{x}^{\prime}\right|}\right) d \mathbf{x}^{\prime} \quad \text { in } D_{ \pm}
$$

with the following properties.

$$
w(\mathbf{x})= \begin{cases}\phi_{+} & \mathbf{x} \text { in } D_{+} \\ \phi_{-} & \mathbf{x} \text { in } D_{-}\end{cases}
$$

and $\mu=\phi_{+}-\phi_{-}$on $S$. The potential $\phi_{+}$vanishes at infinity, which means there is no far field flow. If a far field flow is present, it must be added separately. In our study, we assume there is no far field flow.

Define

$$
\phi=\frac{1}{2}\left(\phi_{+}+\phi_{-}\right) \quad \text { on } S .
$$

Then from potential theory, we have

$$
\phi(\mathbf{x})=-\frac{1}{4 \pi} \oint_{S} \mu\left(\mathbf{x}^{\prime}\right) \mathbf{n}\left(\mathbf{x}^{\prime}\right) \cdot \nabla_{\mathbf{x}^{\prime}}\left(\frac{1}{\left|\mathbf{x}-\mathbf{x}^{\prime}\right|}\right) d \mathbf{x}^{\prime} \quad \text { on } S .
$$

As a consequence, the motion of the sheet is given by

$$
\mathbf{X}_{t}(\alpha, \beta, t)=\nabla \phi
$$

The evolution equation for $\mu$ is obtained as follows. First we determine the rate of change of the potential along the trajectory of a surface particle moving according to 
(5). By differentiating $\phi_{ \pm}(\alpha, \beta, t)=\phi_{ \pm}(\mathbf{X}(\alpha, \beta, t), t)$ with respect to time, keeping the surface coordinates $\alpha$ and $\beta$ fixed, we obtain

$$
\frac{\partial \phi_{ \pm}}{\partial t}=\left.\frac{\partial \phi_{ \pm}}{\partial t}\right|_{\mathbf{x}}+\mathbf{u} \cdot \nabla \phi_{ \pm}
$$

The rate of change of the potential at a fixed point in space can be obtained by integrating (1):

$$
\left.\frac{\partial \phi}{\partial t}\right|_{\mathbf{x}}=-\frac{1}{2} \mathbf{u} \cdot \mathbf{u}-p+c
$$

where $c$ is a constant. Upon substituting the result into (12), we obtain

$$
\frac{\partial \phi_{ \pm}}{\partial t}=\left(\mathbf{u}-\frac{1}{2} \mathbf{u}_{ \pm}\right) \cdot \mathbf{u}_{ \pm}-p_{ \pm}+c_{ \pm} .
$$

By subtracting the rate of change of the potential on either side of the sheet, we find

$$
\frac{\partial \mu}{\partial t}=\left(\mathbf{u}-\frac{1}{2} \mathbf{u}_{+}\right) \cdot \mathbf{u}_{+}-\left(\mathbf{u}-\frac{1}{2} \mathbf{u}_{-}\right) \cdot \mathbf{u}_{-}+c_{+}-c_{-} .
$$

The value of the definition of the velocity for the vortex sheet (5) now becomes clear since (15) simplifies to

$$
\mu=\left.\mu\right|_{t=0}+\left(c_{+}-c_{-}\right) t \quad \text { on } S .
$$

Since a dipole that is constant on $S$ induces only constant potentials inside and outside, the contribution $\left(c_{+}-c_{-}\right) t$ has no dynamical significance, and we may assume $c_{+}=c_{-}=0$ without loss of generality. In other words, we may consider $\mu=\mu(\alpha, \beta)$ on $S$ to be independent of time, and given by the initial conditions.

Next, we describe how $\mathbf{u}$ is calculated. We assume that our coordinate system has the property that $\mathbf{X}_{\beta}, \mathbf{X}_{\alpha}$ and $\mathbf{n}$ form a right-hand system with

$$
\mathbf{n}=\frac{\mathbf{X}_{\beta} \times \mathbf{X}_{\alpha}}{\left|\mathbf{X}_{\beta} \times \mathbf{X}_{\alpha}\right|}
$$

The Greek subscripts refer to differentiation with respect to the surface coordinates. We take the gradient of $w$ as given by (7) and "integrate by parts" over the vortex sheet. Then we take the average of the limiting values on either side of the sheet to obtain

$$
\mathbf{u}=-\frac{1}{4 \pi} \oiint_{S}\left(\mu_{\beta}^{\prime} \mathbf{X}_{\alpha}^{\prime}-\mu_{\alpha}^{\prime} \mathbf{X}_{\beta}^{\prime}\right) \times\left(\frac{\mathbf{X}-\mathbf{X}^{\prime}}{\left|\mathbf{X}-\mathbf{X}^{\prime}\right|^{3}}\right) d \alpha^{\prime} d \beta^{\prime}
$$


We have introduced the notation $\mu^{\prime}=\mu\left(\alpha^{\prime}, \beta^{\prime}\right)$, etc., for convenience. This equation is the same as that obtained from the Biot-Savart law [15].

We obtain a different expression for $\mathbf{u}$ by differentiating $\phi$ (see (11)). In particular,

$$
\mathbf{u}=\frac{\partial \phi}{\partial n} \mathbf{n}+\phi_{\alpha} \frac{\mathbf{n} \times \mathbf{X}_{\beta}}{\left|\mathbf{X}_{\alpha} \times \mathbf{X}_{\beta}\right|}+\phi_{\beta} \frac{\mathbf{X}_{\alpha} \times \mathbf{n}}{\left|\mathbf{X}_{\alpha} \times \mathbf{X}_{\beta}\right|}
$$

The simplest way to determine $\partial \phi / \partial \mathbf{n}$ is through the associated vector potential $\mathbf{A}$ which has the following integral representation,

$$
\mathbf{A}(\mathbf{x})=\frac{1}{4 \pi} \oint_{S} \mu\left(\mathbf{x}^{\prime}\right) \mathbf{n}\left(\mathbf{x}^{\prime}\right) \times \nabla_{\mathbf{x}^{\prime}}\left(\frac{1}{\left|\mathbf{x}-\mathbf{x}^{\prime}\right|}\right) d \mathbf{x}^{\prime} \quad \text { on } S .
$$

Specifically,

$$
\begin{aligned}
\frac{\partial \phi}{\partial n} & =\mathbf{n} \cdot(\nabla \times \mathbf{A}) \\
& =\left[\left(\mathbf{A} \cdot \mathbf{X}_{\alpha}\right)_{\beta}-\left(\mathbf{A} \cdot \mathbf{X}_{\beta}\right)_{\alpha}\right] \frac{1}{\left|\mathbf{X}_{\alpha} \times \mathbf{X}_{\beta}\right|}
\end{aligned}
$$

Notice that once $\phi$ and $\mathbf{A}$ have been computed through (10) and (20), we differentiate them with respect to the surface coordinates only to obtain the velocity components in (19). This then provides an alternate approach to computing the motion of the vortex sheet.

The advantage of using the surface integrals (10) and (19) is that the following identities:

$$
\begin{gathered}
-\frac{1}{4 \pi} \oint \mathbf{n}\left(\mathbf{x}^{\prime}\right) \cdot \nabla_{\mathbf{x}^{\prime}}\left(\frac{1}{\left|\mathbf{x}-\mathbf{x}^{\prime}\right|}\right) d \mathbf{x}^{\prime}=\frac{1}{2}, \\
\frac{1}{4 \pi} \oint \mathbf{n}\left(\mathbf{x}^{\prime}\right) \times \nabla_{\mathbf{x}^{\prime}}\left(\frac{1}{\left|\mathbf{x}-\mathbf{x}^{\prime}\right|}\right) d \mathbf{x}^{\prime}=0
\end{gathered}
$$

may be used to reduce the singularities in their integrands [9]. For example, A can be rewritten as

$$
\mathbf{A}(\mathbf{x})=\frac{1}{4 \pi} \oint\left(\mu\left(\mathbf{x}^{\prime}\right)-\mu(\mathbf{x})\right) \mathbf{n}\left(\mathbf{x}^{\prime}\right) \times \nabla_{\mathbf{x}^{\prime}}\left(\frac{1}{\left|\mathbf{x}-\mathbf{x}^{\prime}\right|}\right) d \mathbf{x}^{\prime} \quad \text { on } S .
$$

We will show specifically in the case of axi-symmetric flow how the singularities in the integrands have been reduced. 
For an axi-symmetric vortex sheet, we may represent the sheet location as

$$
\mathbf{X}=r(\alpha, t) \mathbf{e}_{r}+z(\alpha, t) \mathbf{e}_{z}
$$

using the cylindrical coordinates $(r, z)$. The motion of the sheet is assumed independent of the azimuthal angle $\beta=\theta$. As noted before [15] for motion without swirl, the Biot-Savart integral (18) becomes

$$
\begin{aligned}
& r_{t}=\frac{1}{4 \pi r} \oint \mu_{\alpha}^{\prime}\left(z^{\prime}-z\right) B_{0}\left(\alpha, \alpha^{\prime}\right)\left[F(k)+B_{1}\left(\alpha, \alpha^{\prime}\right) E(k)\right] d \alpha^{\prime}, \\
& z_{t}=\frac{1}{4 \pi} \oint \mu_{\alpha}^{\prime} B_{0}\left(\alpha, \alpha^{\prime}\right)\left[F(k)+B_{2}\left(\alpha, \alpha^{\prime}\right) E(k)\right] d \alpha^{\prime},
\end{aligned}
$$

where $F(k)$ and $E(k)$ are the complete elliptic integrals

$$
F(k)=\int_{0}^{\frac{\pi}{2}}\left(1-k^{2} \cos ^{2}(\beta)\right)^{-\frac{1}{2}} d \beta, \quad E(k)=\int_{0}^{\frac{\pi}{2}}\left(1-k^{2} \cos ^{2}(\beta)\right)^{\frac{1}{2}} d \beta
$$

of the first and second kind respectively, with

$$
k^{2}=\frac{4 r r^{\prime}}{\left(z-z^{\prime}\right)^{2}+\left(r+r^{\prime}\right)^{2}} .
$$

The other quantities are

$$
\begin{aligned}
& B_{0}\left(\alpha, \alpha^{\prime}\right)=\frac{2}{\left(\left(z-z^{\prime}\right)^{2}+\left(r+r^{\prime}\right)^{2}\right)^{1 / 2}}, \\
& B_{1}\left(\alpha, \alpha^{\prime}\right)=-\frac{\left(z^{\prime}-z\right)^{2}+r^{\prime 2}+r^{2}}{\left(z-z^{\prime}\right)^{2}+\left(r-r^{\prime}\right)^{2}}, \\
& B_{2}\left(\alpha, \alpha^{\prime}\right)=\frac{r^{\prime 2}-r^{2}-\left(z^{\prime}-z\right)^{2}}{\left(z-z^{\prime}\right)^{2}+\left(r-r^{\prime}\right)^{2}} .
\end{aligned}
$$

Now our notation reflects the dependency on only one surface coordinate, $r^{\prime}=r\left(\alpha^{\prime}\right)$, etc.

In the alternate formulation, (19) becomes

$$
\begin{aligned}
& r_{t}=\left(\phi_{\alpha} r_{\alpha}-\frac{\psi_{\alpha}}{r} z_{\alpha}\right) \frac{1}{s_{\alpha}^{2}}, \\
& z_{t}=\left(\phi_{\alpha} z_{\alpha}+\frac{\psi_{\alpha}}{r} r_{\alpha}\right) \frac{1}{s_{\alpha}^{2}},
\end{aligned}
$$


where $s_{\alpha}^{2}=r_{\alpha}^{2}+z_{\alpha}^{2}$ and $\psi=r \mathbf{A} \cdot \mathbf{e}_{\theta}$ is a pseudo-streamfunction. Note that $\mathbf{A}$ has only one non-vanishing component. By using $(22,23)$, we may express the potential function (10) as

$$
\phi=\frac{1}{4 \pi} \oint\left(\mu^{\prime}-\mu\right) B_{0}\left(\alpha, \alpha^{\prime}\right)\left[z_{\alpha}^{\prime} F(k)+C_{1}\left(\alpha, \alpha^{\prime}\right) E(k)\right] d \alpha^{\prime}+\frac{1}{2} \mu,
$$

and the pseudo-streamfunction (obtained from (20)) as

$$
\psi=\frac{1}{4 \pi} \oint\left(\mu^{\prime}-\mu\right) B_{0}\left(\alpha, \alpha^{\prime}\right)\left\{\left[r_{\alpha}^{\prime} r^{\prime}-z_{\alpha}^{\prime}\left(z-z^{\prime}\right)\right] F(k)+C_{2}\left(\alpha, \alpha^{\prime}\right) E(k)\right\} d \alpha^{\prime},
$$

where

$$
\begin{gathered}
C_{1}=-\frac{\left(z-z^{\prime}\right)\left[z_{\alpha}^{\prime}\left(z-z^{\prime}\right)-2 r^{\prime} r_{\alpha}^{\prime}\right]+\left(r^{2}-{r^{\prime}}^{2}\right) z_{\alpha}^{\prime}}{\left(z-z^{\prime}\right)^{2}+\left(r-r^{\prime}\right)^{2}} \\
C_{2}=\frac{\left(z-z^{\prime}\right)\left(\left[z_{\alpha}^{\prime}\left(z-z^{\prime}\right)-r_{\alpha}^{\prime} r^{\prime}\right]\left(z-z^{\prime}\right)+z_{\alpha}^{\prime}\left(r^{2}+{r^{\prime}}^{2}\right)\right)+\left(r^{2}-{r^{\prime}}^{2}\right) r_{\alpha}^{\prime} r^{\prime}}{\left(z-z^{\prime}\right)^{2}+\left(r-r^{\prime}\right)^{2}} .
\end{gathered}
$$

In order to design good numerical methods for the integrals, we must understand the nature of the singularities in the integrands. First, we consider the asymptotic behavior of the integrands in $(26,27)$ when the integration variable $\alpha^{\prime}$ is close to the field point at $\alpha$. For $r \equiv r(\alpha) \neq 0$, the leading order terms of the integrands are

$$
-\frac{2 r \mu_{\alpha} z_{\alpha}}{s_{\alpha}^{2}\left(\alpha^{\prime}-\alpha\right)} \quad \text { and } \quad \frac{2 \mu_{\alpha} r_{\alpha}}{s_{\alpha}^{2}\left(\alpha^{\prime}-\alpha\right)}
$$

respectively. Clearly, the integrands have pole singularities $1 /\left(\alpha^{\prime}-\alpha\right)$ except right on the axis.

In the other formulation, the integrands in $(35,36)$ exhibit the following asymptotic behavior

$$
-\frac{z_{\alpha} \mu_{\alpha}}{r}\left(\alpha^{\prime}-\alpha\right) \ln \left|\alpha-\alpha^{\prime}\right| \quad \text { and } \quad-2 \mu_{\alpha} r
$$

respectively. Therefore, these integrands are continuous even though they have discontinuous derivatives. This information will prove very valuable in the design of effective numerical methods.

\section{Numerical Methods}

We describe the numerical methods to evaluate the velocity of the vortex sheet in three parts: evaluation of the complete elliptic integrals of the first and second kind; 
adaptive numerical quadratures; and their specific application to the vortex sheet.

\subsection{Evaluation of the elliptic integrals}

In ([16], p297), there are recursive formulae for the evaluation of both $F(k)$ and $E(k)$.

$$
F=\frac{\pi}{2}\left(1+G_{1}\right)\left(1+G_{2}\right)\left(1+G_{3}\right) \ldots, \quad E=F\left(1-\frac{k^{2}}{2} H\right)
$$

where

$$
H=1+\frac{G_{1}}{2}\left(1+\frac{G_{2}}{2}\left(1+\frac{G_{3}}{2}(\ldots) \ldots\right) \ldots\right),
$$

and $G_{i}$ is given by the recursion,

$$
G_{0}=k, \quad G_{i}=\frac{1-\sqrt{1-G_{i-1}^{2}}}{1+\sqrt{1-G_{i-1}^{2}}} .
$$

This method is very robust computationally. However, the number of iterations for a given tolerance depends on $k$. In general, more iterations are needed when $k$ is close to 1 .

The other widely-used technique for the computation of $E$ and $F$ is a polynomial approximation. From ([16], p297) and ([17], p170), we know

$$
F=\ln (4)+\sum_{j=1}^{m} a_{j} \zeta^{j}+\left(\frac{1}{2}+\sum_{j=1}^{m} b_{j} \zeta^{j}\right) \ln \left(\frac{1}{\zeta}\right)+\operatorname{error}_{1}(k)
$$

and

$$
E=1+\sum_{j=1}^{m} c_{j} \zeta^{j}+\left(\sum_{j=1}^{m} d_{j} \zeta^{j}\right) \ln \left(\frac{1}{\zeta}\right)+\operatorname{error}_{2}(k)
$$

where $\zeta=1-k^{2}$. The polynomial coefficients are obtained via least-square fits. The sizes of the two error terms depend on $m$. For example, to guarantee errors of less than $10^{-8}$ we need $m=4$ ([17], p170) ([18], p591), a very common choice. To guarantee errors of less than $10^{-21}$, we need $m=15$, which is still not excessive. 


\subsection{Adaptive Quadratures}

Gaussian quadrature is one of the most popular methods for numerical integration because of its high accuracy. However, the standard Gaussian quadrature lacks the nature of progressiveness, i.e. a Gaussian rule can not be generated by adding new points to another Gaussian rule, and it has to be reconstructed. This is not suitable for adaptive strategy.

In [19], Kronrod shows that an $n$-point Gaussian rule may be augmented by a further set of $n+1$ abscissae to yield a rule which integrates exactly a polynomial of degree $3 n+1$ for $n$ even and $3 n+2$ for $n$ odd. In the extended rule, there are extra $n+1$ weights and abscissae. While the original Gaussian abscissae remain unchanged, their weights must be reset. Patterson [20] extends this technique by considering the argumentation of an $n$-point formula by $p$ points. There are tables containing the standard abscissae and weights in [19].

In this study, we use a 7-15 point Gauss-Kronrod quadrature. We bisect the integration intervals and estimate the error of the numerical integration over each interval by measuring the difference between the 7-point Gauss quadrature and 15point Kronrod rule. A globally adaptive strategy is to bisect the interval with the largest error estimate until the error is less than a prescribed tolerance. We used the freely available software package Quadpack [21] which implements this strategy.

Clenshaw-Curtis Quadrature [22] is based on the expansion of the integrand as a series of Chebyshev polynomials. The method is naturally adaptive since it adjusts the number of terms to reach a specified level of accuracy. For integrands with weight functions which cause a slow rate of convergence, Piessens and Brander [23] developed a modified Clenshaw-Curtis quadrature through the computation of modified Chebyshev moments. In this study, we are interested in the computation of the integral with the following weight [21],

$$
\int_{0}^{1} f\left(\alpha^{\prime}\right) \ln \left(\alpha^{\prime}\right) d \alpha^{\prime}
$$

This weight function is one of the choices available in Quadpack which contains the software necessary to perform the modified Clenshaw-Curtis quadrature. 


\subsection{Application to the Dipole Distribution}

A convenient choice for the surface coordinate is to use the angle subtended at the center of the enclosed region by the axis of symmetry and the vector to a field point on the initial location of the surface. For the studies reported in this paper, the initial surface is a sphere and $\alpha$ is the polar angle measured from the bottom pole. Thus $\alpha=0$ will mark the bottom pole, while $\alpha=\pi$ will mark its top.

We approximate the closed surface by a set of points along the contour in the $(r, z)$ meridional plane $\left(r_{i}, z_{i}\right) \equiv\left(r\left(\alpha_{i}\right), z\left(\alpha_{i}\right)\right)$ for $i=1, \ldots, N$, where $\alpha_{i}=i \pi /(N-1)$. The equations $(33,34)$ are then enforced at these points. This first requires the computation of $\phi_{i}$ and $\psi_{i}$. Although the integrands in $(35,36)$ are continuous when $\alpha=\alpha^{\prime}$, their derivatives are not. So we split the integrals into two parts: one integrated from 0 to $\alpha_{i}$, and the other one from $\alpha_{i}$ to $\pi$. Then we apply the adaptive Gauss-Kronrod quadrature to each of the two integrals. Because Gauss-Kronrod quadrature is a open quadrature, we do not need to evaluate the integrands at the integration limits. In particular, we do not need to worry about the indeterminate form of the integrand when $\alpha=\alpha^{\prime}$.

To evaluate the integrands, we also need values for the elliptic integrals and derivatives of $r$ and $z$. The elliptic integrals are determined through the recursion (41-43). Depending on the accuracy we require for the integration, we set a tolerance for the recursion for the elliptic integrals. To determine the derivatives of $r$ and $z$ we expand them in a discrete fourier cosine series and differentiate the series. This can be done in $O(N)$ operations through the Fast Fourier Transform. Similarly we can determine the derivatives of $\phi$ and $\psi$ which then give us the velocities of the points on the sheet. To obtain values of quantities at points other than the collocation points, we use quintic splines. Because of the symmetries in a closed, axi-symmetric surface, the end point conditions for the splines are easily specified. We call this procedure Method 1.

In Method II, we replace the elliptic integral of the first and second kind by the 
approximations $(44,45)$. The two integrals $(35,36)$ then take the form,

$$
\int_{0}^{\pi} f\left(\alpha, \alpha^{\prime}\right) d \alpha^{\prime}+\int_{0}^{\alpha} g_{1}\left(\alpha, \alpha^{\prime}\right) \ln \left(\alpha-\alpha^{\prime}\right) d \alpha^{\prime}+\int_{\alpha}^{\pi} g_{2}\left(\alpha, \alpha^{\prime}\right) \ln \left(\alpha^{\prime}-\alpha\right) d \alpha^{\prime}
$$

where $f, g_{1}$, and $g_{2}$ are smooth functions of $\alpha$ and $\alpha^{\prime}$. We use the Gauss-Kronrod quadrature on the first integral in (47), and the Modified Clenshaw-Curtis quadratue on the second and third integrals in (47) respectively. As in method I, derivatives are obtained through the use of a Fourier cosine series, and interpolation is conducted with quintic splines.

Once the velocity components have been determined by Method I or II, we advance the location of the vortex sheet by applying a fourth-order Adams-Moulton predictor-corrector on $(33,34)$. Starting values for the predictor-corrector are computed by a standard fourth-order Runge-Kutta method.

\section{Numerical Tests of Methods}

Our first series of tests are performed without using adative quadrature. This provides us with data by which we can compare the effectiveness of adaptive quadrature. We explore three non-adaptive strategies to compute the velocity $\left(r_{t}, z_{t}\right)$ of a vortex sheet. In Method A, we apply the six-point Gaussian quadrature in each sub-interval $\left(\alpha_{i}, \alpha_{i+1}\right)$ of the Biot-Savart integrals $(26,27)$. In Method B, we follow previous work [10] by summing the integrands of the Biot-Savart integrals at all collocation points except the field point, then adding the axi-symmetric Van der Vooren corrections to the sum. In Method C, we apply the six-point Gaussian quadrature in each sub-interval $\left(\alpha_{i}, \alpha_{i+1}\right)$ of the integrals $(35,36)$. In all three cases, we use the Fourier series to determine derivatives, and interpolation is performed through the use of quintic splines. The two elliptic integrals are computed through the recursive formulae $(41,42,43)$.

We use the instantaneous velocity field of a uniform flow past a sphere as a test calculation. The parametrization of the surface of the sphere and the dipole distribution is

$$
r(\alpha)=\sin (\alpha) \quad z(\alpha)=-\cos (\alpha) \quad \mu(\alpha)=\cos (\alpha)
$$


where $\alpha \in[0, \pi]$. The velocities of the vortex sheet are

$$
r_{t}=\frac{\sin (2 \alpha)}{4} \quad z_{t}=-\left(\frac{5}{12}+\frac{1}{4} \cos (2 \alpha)\right)
$$

We measure the error as the maximum difference between the exact velocity (49) and the numerically calculated one. In Figure 1, we plot the errors for the three different methods when $N=65$. Method C, based on the use of the vector potential, gives the best accuracy, but all three methods lose accuracy when $\alpha$ is near the two poles. The cause of the difficulty is the particular behavior of the integrand for a field point near the axis of symmetry [1], [11]. The error profile has a boundary layer inside of which the error only decreases linearly with the spacing between the collocation points [14]. We confirm this behavior in Figure 2, which shows the variation of the maximum error with the number of collocation points. The decrease is only linear. By linear extrapolation, the error will be about $10^{-7}$ and $10^{-10}$ with $N=2^{20}$ for Method B and Method C, respectively. The cost is prohibitive to achieve the levels of accuracy rquired to study singularity formation on vortex sheets.

We turn now to the study of the two adaptive methods. For all the results reported here (including the next section), we make sure that the errors in the computation of the elliptic integrals are less than $\epsilon_{E}=10^{-14}$. For the adaptive Gauss-Kronrod quadrature (Method A), we use the recursive formulae $(41,42,43)$ until the difference in the iterates are less than $\epsilon_{E}$. For the adaptive Clenshaw-Curtis quadrature (Method B), we find that $m=8$ ensures the polynomial approximations to the elliptic functions are accurate to at least $\epsilon_{E}$. Also, we set a tolerance requirement $\epsilon_{I}$ for the calculation of the various integrals. This tolerance is simply passed through a subroutine call to the software package Quadpack. Figure 3 presents the errors of the two adaptive methods as a function of $\alpha$ when $N=65$ and $\epsilon_{I}=10^{-12}$. In contrast to the non-adaptive methods, the errors are nearly uniform for all $\alpha$. The errors are very close to the specified tolerance for the calculation of the integrals. Don't forget that $\phi$ and $\psi$ must be differentiated numerically to determine the velocities on the vortex sheet, and there will be some error incurred as a result. 
In order to compare the cost between these two methods, we count the number of integrand evaluations for each method, which is the dominant cost in computing the integrals. In Figure 4, we plot the average number of integrand evaluations per collocation point as a function of the error. We show the results for both $N=65$ and $N=129$. There are several points that the these results illustrate. First, consistent with the results of the previous test, it requires many quadrature points to obtain even moderate accuracy. By using an adaptive quadrature we insert the quadrature points through interpolation just where they are needed. Without adaption, we would have to introduce many more collocation points, driving up the cost of numerical integration exorbitantly. Note furthermore that the number of integrand evaluations is relatively insensitive to the number of collocation points. This means that the errors in using $N=65$ and $N=129$ points to represent the interface are much smaller than the errors caused by the numerical integration. In other words, there is no need to have more collocation points. Finally, we note that Method II is more efficent than Method I, and both methods are much more efficient than Method C.

\section{Evolution of Vortex Sheet}

We follow previous work [13], [14] in using (48) as the initial condition. This initial condition corresponds to a uniform potential flow past a solid sphere which is instantaneously dissolved at $t=0$. The vortex sheet at the boundary of the sphere is then allowed to evolve freely.

Since vortex sheet motion is ill-posed, it is necessary to control the growth of round-off errors to avoid rapid contamination of the profile long before singularity formation. In studies of two-dimensional motion of vortex sheets, a Fourier filter is frequently used [24]: the reliability of the filter has been checked with arbitrary precision calculations [25]. At each time step, we calculate the Fourier spectrum of the location of the vortex sheet and set all amplitudes below a filter level $\epsilon_{F}$ to zero.

In Figure 5, the profiles of the axi-symmetric vortex sheet are shown at different times. The results are obtained by Method II using $\epsilon_{F}=\epsilon_{I}=10^{-12}, N=257$, and 
the time step $\triangle t=0.001$. We establish the accuracy in our results by a resolution study. First, we consider the influence of the number of collocation points $N$ which represent the interface. We treat $N=257$ as "exact", and use the maximal difference between this "exact" solution and other smaller $N$ as an estimate of the error. We plot the error for $N=9,17,33,65,129$ in Figure 6. Here, the time step is $\triangle t=0.001$. There are three major sources of error due to the spatial discretization: there is an $O\left(N^{-6}\right)$ error caused by the use of quintic splines for interpolation; there is an $O(\exp (-c N))$ error in determining the derivatives of $\phi$ and $\psi$ by their Fourier series ( $c$ is some constant); and there is an error produced by the evaluation of the integrals. In general, if the errors in evaluating the integrals are small enough compared with the other two errors, the dominant error should be $O\left(N^{-6}\right)$. Specifically, the error should decrease by a factor of 64 whenever $N$ is doubled. This is evident in Figure 6. The errors uniformly decrease by a facor close to 64 as the number of collocation points is increased from $N=9$ to $N=65$. For $N=65$ and $N=129$, the errors are initially limited by the choices $\epsilon_{I}=\epsilon_{F}=10^{-12}$, but as the errors become larger than $10^{-12}$, the accuracy for $N=129$ is better than for $N=65$.

Next we consider the errors caused by the temporal discretization. Similar to the spatial resolution study, we take the results for $\Delta t=0.00025$ as "exact", and use the difference with the results for larger $\Delta t$ as estimates of the errors. Here, we pick $N=129$. Since the Adams-Moulton Predictor-Corrector method has an error of $O\left((\triangle t)^{4}\right)$, we should obtain an improvement in accuracy of a factor of 16 whenever $\Delta t$ is halved. In Figure 7, the errors are plotted as functions of time for $\triangle t=0.04,0.002,0.001,0.005,0.0025,0.001,0.0005$.

Except at very early times, the errors uniformly decrease with a factor about 16 as $\Delta t$ decreases to 0.0025 . For smaller values of $\Delta t$, the errors become smaller than the effects of the spectral filter $\left(\epsilon_{F}=10^{-12}\right)$, and no further improvement is possible without changing the various tolerance requirements and the filter level. Consequently, $\triangle t=0.001$ gives the best accuracy for our choice of numerical parameters. Most important is the fact that no smoothing other than the spectral filter to control round-off errors is needed for numerical stability. Highly accurate 
evaluations of the integrals give numerically stable methods, an observation noted in two dimensional motion [26], [27].

We turn now to comparison of the performance of Method I and Method II. From the resolution study, we find that $N=129$ and $\triangle t=0.001$ gives levels of accuracy close to $\epsilon_{I}=10^{-12}$. So we choose this $N$ and $\Delta t$ for the comparison of Method I and Method II. We compute solutions for both methods with three different choices of $\epsilon_{I}=10^{-8}, 10^{-10}, 10^{-12}\left(\epsilon_{I}=\epsilon_{F}\right)$. In Figure 8, we show the maximal differences of the solutions between these two methods. As seen in the plot, the differences are comparable with the size of $\epsilon_{I}$ at early times, and increase slightly as time advances. The general tendency of the curves is similar to those shown in the resolution study for Method II. We conclude that the two methods are comparable in terms of achieving accuracy. However, Method II is superior in terms of efficiency. This is evident in Table 1, where the computing time for both methods is listed for different $\epsilon_{I}$. Roughly speaking, Method II is about four times faster than Method I. Even so, both methods are very effective considering the accuracy they achieve.

We conclude this section by providing some evidence of the formation of a curvature singularity in the vortex sheet. In Figure 9, we show $r_{\alpha \alpha}$ as a function of $\alpha$ at times close to when the magnitude of the curvature grows rapidly. This behavior is quite typical of that seen in singularity formation in two dimensional motion of vortex sheets [7]. This singular behavior explains why the solutions gradually lose their accuracy as time approaches the "singularity time". A detailed study of this singular behavior will be presented in [28].

\section{Conclusions}

Many quadrature points are needed to obtain accurate boundary integrals for the axi-symmetric motion of a vortex sheet. If a fixed quadrature rule is used, this also requires many collocation points, driving the cost to prohibitive levels. By using adaptive quadratures, the number of quadrature points can be made independent of the number of collocation points, leading to very efficient techniques for the nu- 


\begin{tabular}{|c|c|c|c|}
\hline$\epsilon_{I}$ & $10^{-8}$ & \multicolumn{1}{|c|}{$10^{-10}$} & \multicolumn{1}{c|}{$10^{-12}$} \\
\hline Method I & 73 mins & 115 mins & 160 mins \\
Method II & 19 mins & 26 mins & 35 mins \\
\hline
\end{tabular}

Table 1: Running Time on SGI Indigo2 Impact 10000

merical study of vortex sheet motion. We anticipate similar results for boundary integral methods to track free surface motion in axi-symmetric geometry.

\section{Acknowledgments}

We would like to thank Professor D. W. Moore for many valuable discussions and providing us with his code for Method B. We also benefiited from several conversations with Monika Nitsche. Some of the computations were performed at the Ohio Supercomputer Center whose grant of computing resources is gratefully acknowledged. 


\section{References}

[1] G. R. Baker, D. I. Meiron and S. A. Orszag. Generalized vortex methods for free-surface flow problems. J. Fluid Mech., 123:477-501, 1982.

[2] C. Pozrikidis. Boundary integral and singularity methods for linearized viscous flow. Cambridge University Press, 1992.

[3] G.R. Baker and D.W. Moore. The rise and distortion of a two-dimensional gas bubble in an inviscid liquid. Phys. Fluids, A1:1451, 1989.

[4] G. R. Baker, D. I. Meiron, and S. A. Orszag. Vortex simulations of the rayleightaylor instability. Phys. Fluids, 23:1485-1490, 1980.

[5] G. R. Baker. Generalized vortex methods for free-surface flows. In R. E. Meyer, editor, Waves on Fluid Interfaces. Academic Press, 1983.

[6] A. Sidi and M. Israeli. Quadrature methods for singular and weakly fredholm integral equations. J. Sci. Comput., 3:201-231, 1988.

[7] M.J. Shelley. A study of singularity formation in vortex sheet motion: a spectrally accurate method. J. Fluid Mech., 1992.

[8] G. R. Baker, R. E. Caflisch and M. Siegel. Singularity formation during Rayleith-Taylor instability. J. Fluid Mech., 1993.

[9] G. R. Baker, D. I. Meiron and S. A. Orszag. Boundary integral methods for axisymmetric and three-dimensional Rayleigh-Taylor instability problems. Physical D, 12:19-31, 1984.

[10] B. Bernadinis and D. W. Moore. A ring-vortex representation of an axisymmetric vortex sheet. In Studies of Vortex Dominated Flows, pages 33-43. Springer-Verlag, 1987.

[11] M. Nitsche. Axi-symmetric vortex sheet motion: accurate evaluation of the principal value integral. Preprint, 1997.

[12] T. S. Lundgren and N. N. Mansour. Oscillations of drops in zero gravity with weak viscous effects. J. Fluid Mech., 1988. 
[13] D. A. Pugh. Development of vortex sheets in Boussinesq flows-formation of singularities. PhD thesis, Imperial College of Science and Technology, 1989.

[14] M. Nitsche. Evolution of a cylindrical and spherical vortex sheet. In Proceeding of second international workshop on vortex flows and related numerical methods, 1995.

[15] R. E. Caflisch and Xiao-Fan Li. Lagrangian theory for 3D vortex sheets with axial or helical symmetry. Transport Theory and Statistical Physics, 21:559-578, 1992.

[16] Paul F. Byrd and Morris D. Friedman. Handbook of Elliptic Integrals for Engineers and Physicists. Springer-Verlag, 1953.

[17] Jr. Cecil Hastings. Approximations for Digital Computers. Princeton University Press, 1955.

[18] Milton Abramowitz and Irene A. Stegun. Handbook of Mathematical Functions. Dover, 1970.

[19] A. S. Kronrod. Nodes and Weights of Quadrature Formulas. Consultants Bureau, New York, 1965.

[20] T. N. Patterson. The optimum addition of points to quadrature formulae. Math. Comp., 22:847-856, 1968.

[21] R. Piessens, E. de Doncker-Kapenga, C. W. Uberhuber, D. K. Kahaner. Quadpack. Springer-Verlag, 1983.

[22] C. Clenshaw and A. R. Curtis. A method for numerical integration on an automatic computer. Numer. Math, 12:197-205, 1960.

[23] R. Piessens and M. Branders. The evaluation and application of some modified moments. BIT, 13:443-450, 1973.

[24] R. Krasny. A study of singularity formation in a vortex sheet by the point vortex approximation. J. Fluid Mech., 167:65-93, 1986. 
[25] J. Ely and G. R. Baker. High precision calculations of vortex sheet motion. $J$. Comp. Phys., 111:275-282, 1994.

[26] A.J. Roberts. A stable and accurate numerical method to calculate the motion of a sharp interface between fluids. IMA J. of Appl. Math., 31:13-28, 1983.

[27] A. Nachbin and G.R. Baker. Stable methods for vortex sheet motion in the presence of surface tension. to appear in SIAM J. Sci. Comp., 1997.

[28] G. R. Baker and Q. Nie. Singularity formation in an axi-symmetric vortex sheet. In Preparation, 1996. 


\section{List of Figure Captions}

1. Errors as functions of $\alpha$ for three non-adaptive methods. Dotted curve: Method A; dash curve: Method B; solid curve: Method C.

2. Log-log plot of the maximum error of each method as a function of $N$. "x": Method A; "o": Method B; "*”: Method C.

3. Errors as functions of $\alpha$ for the two adaptive methods. Solid curve: Method I; dash curve: Method II.

4. Log-log plot of the average number of integrands evaluation per collocation point as functions of the errors. "x": Method I with $N=65$; “*”: Method I with $N=129$; "o": Method II with $N=65$; "+": Method II with $N=129$.

5. Profiles of the vortex sheet at different times.

6. The maximal differences between the solution for $N=257$ and smaller $N$ as functions of time.

7. The maximal differences between the solution for $\triangle t=0.00025$ and for larger $\triangle t$ as functions of time.

8. The maximal difference between the solutions computed by Method I and Method II with different choices of $\epsilon_{I}$ : a) dotted curve: $\epsilon_{a}=10^{-8}$; b) dash curve: $\epsilon_{a}=10^{-10} ;$ c) solid curve: $\epsilon_{a}=10^{-12}$.

9. $r_{\alpha \alpha}$ vs $\alpha$ at $t=1+i \times 0.02(i=1,4)$ and $t=1.095$. 


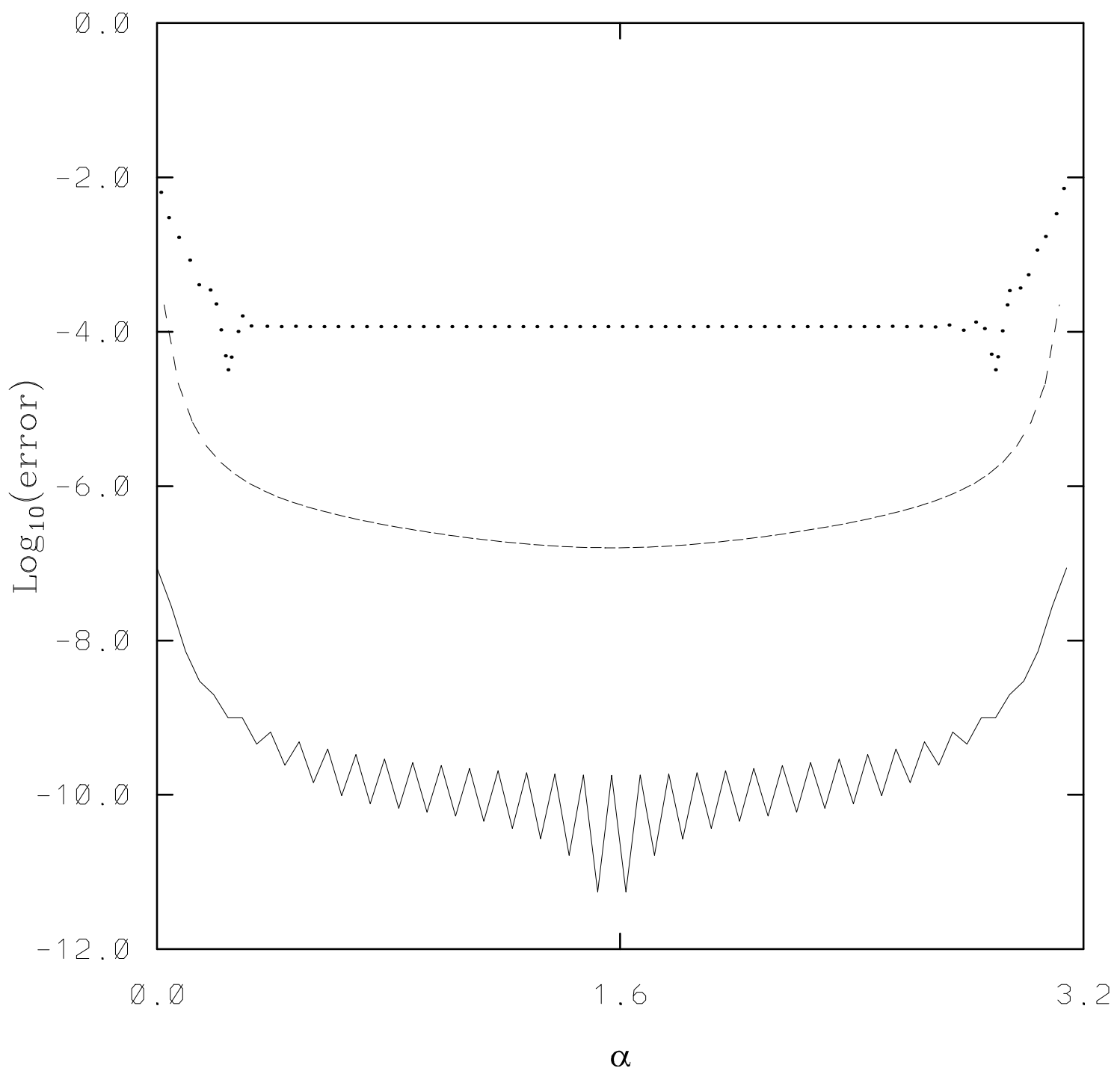

Figure 1: 


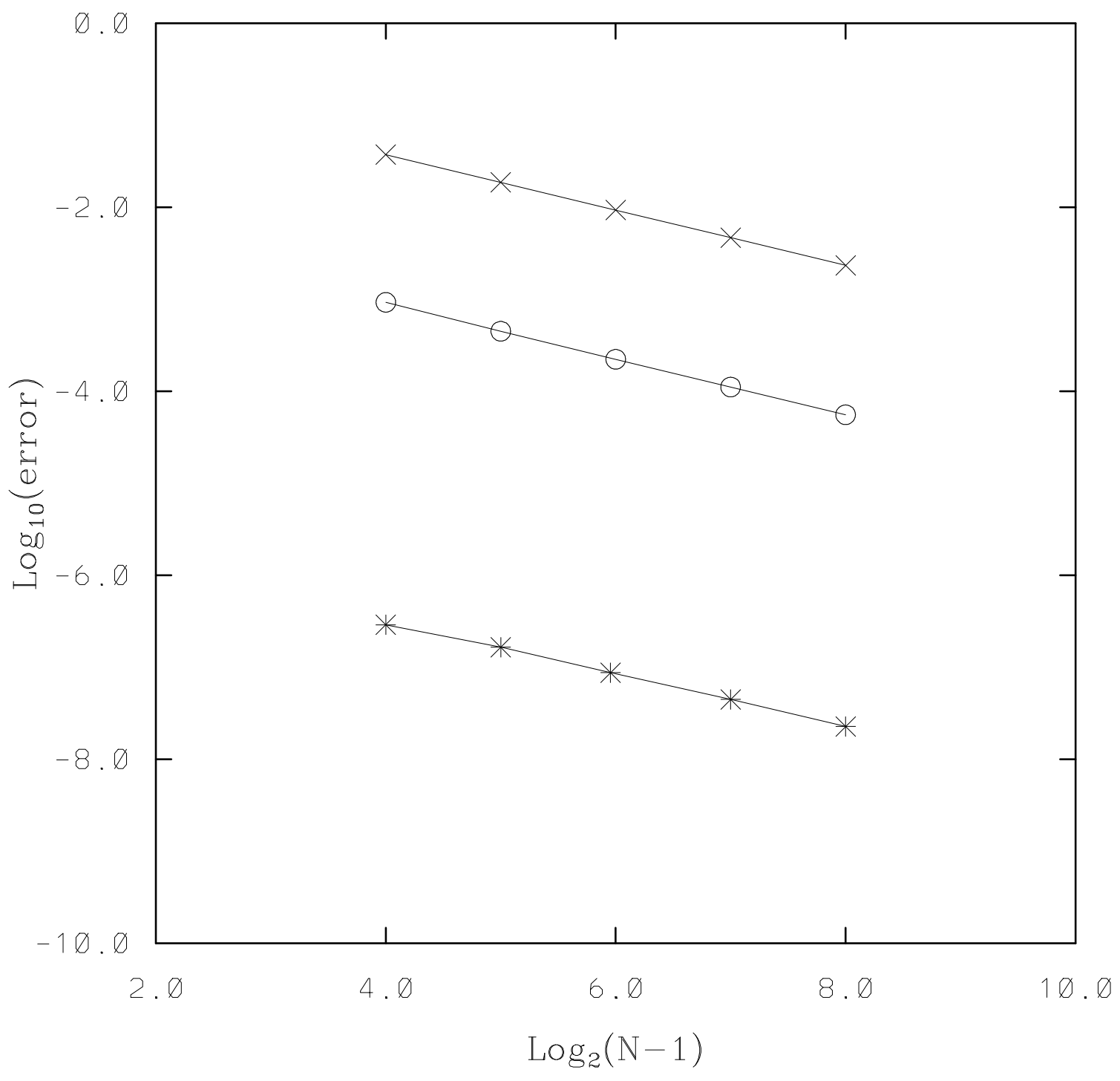

Figure 2: 


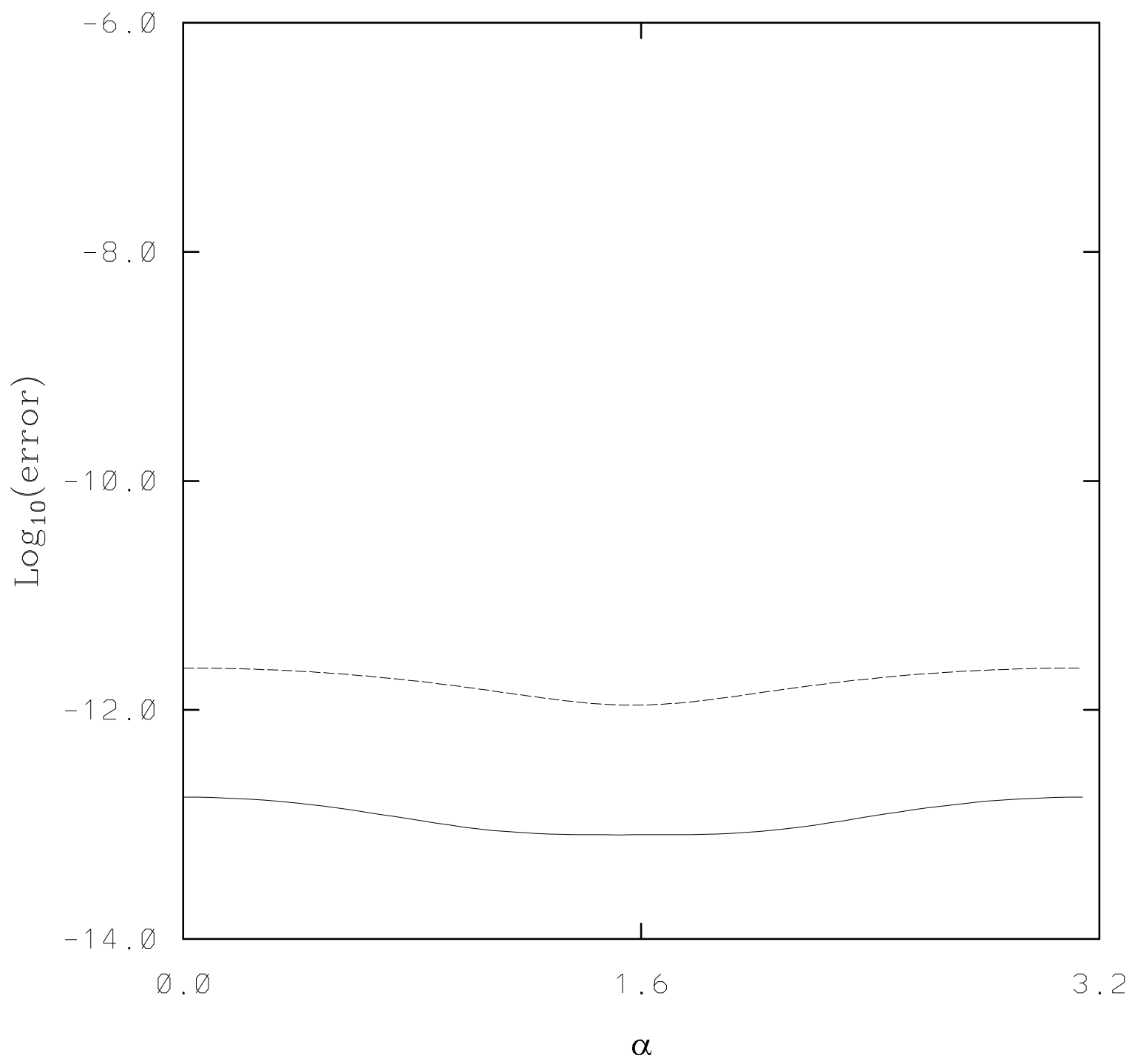

Figure 3: 


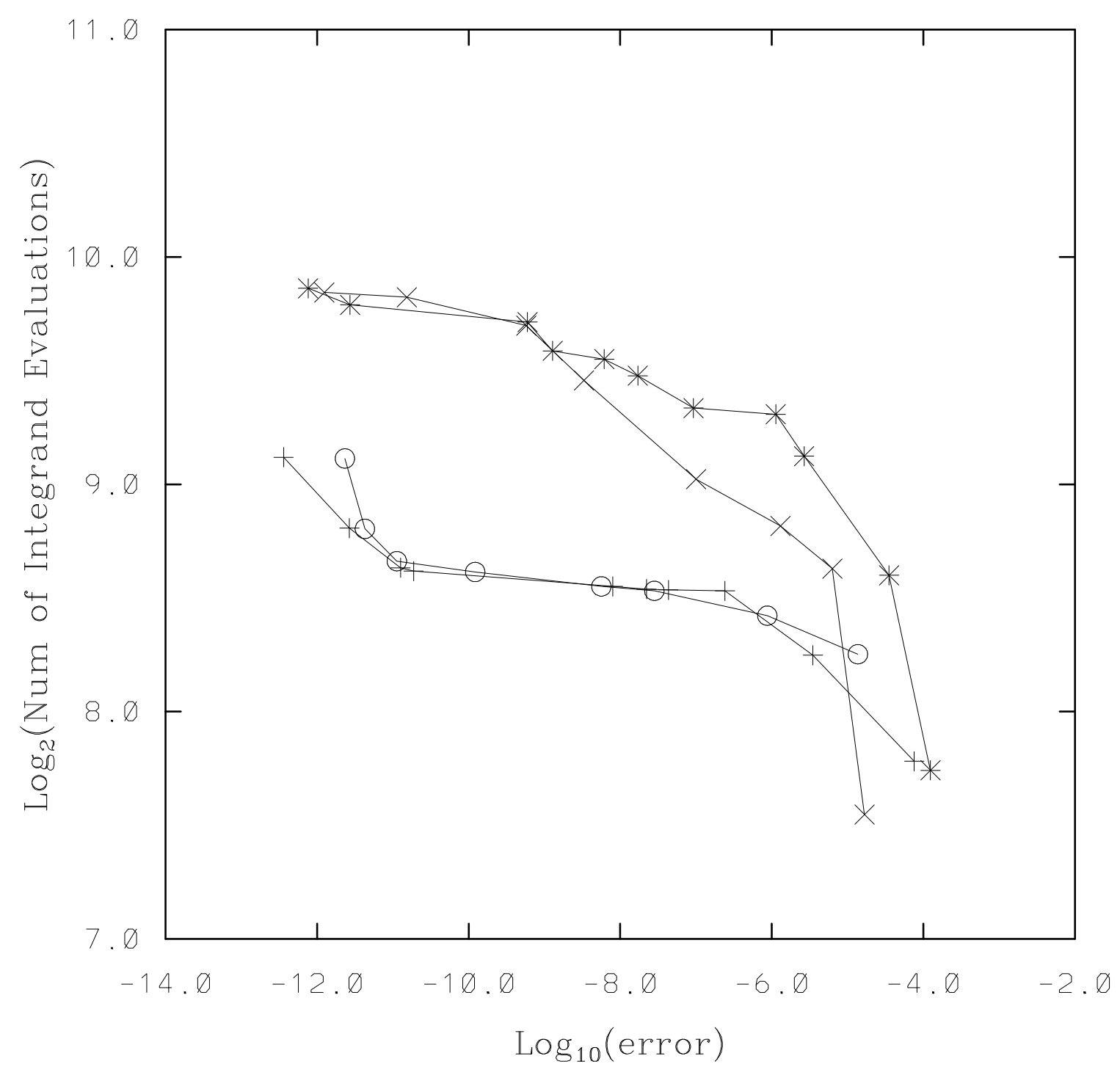

Figure 4: 

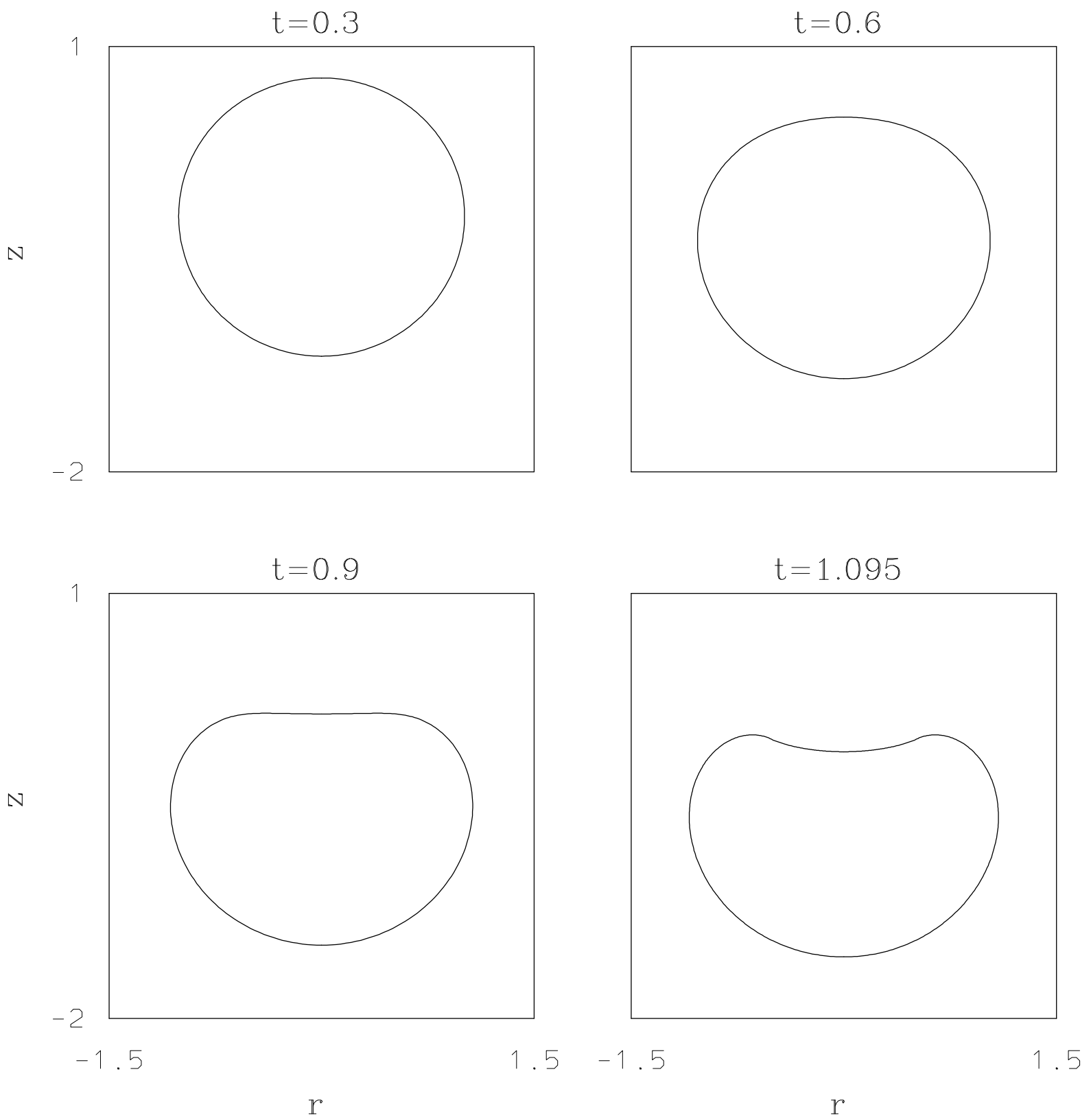

Figure 5: 


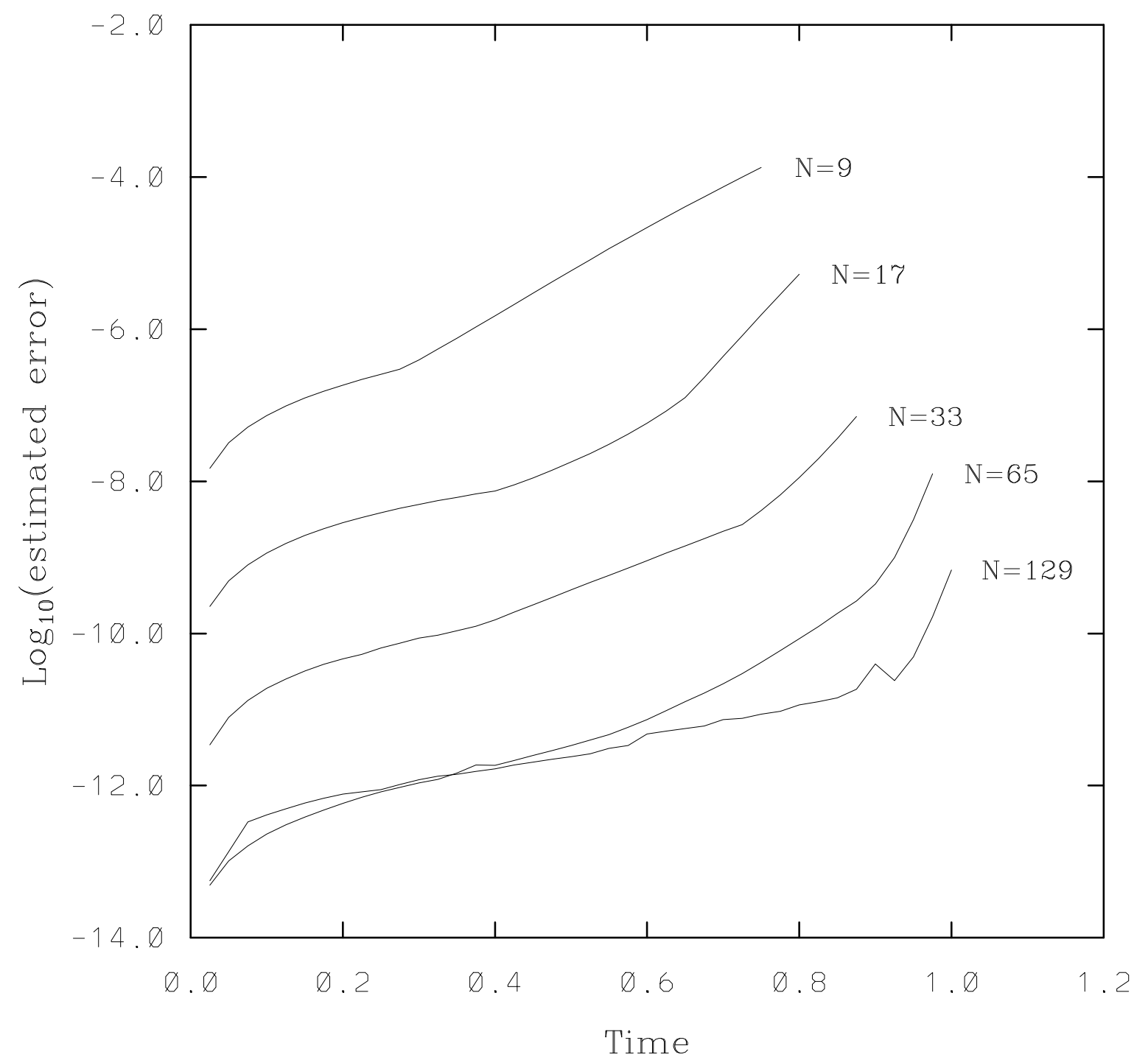

Figure 6: 


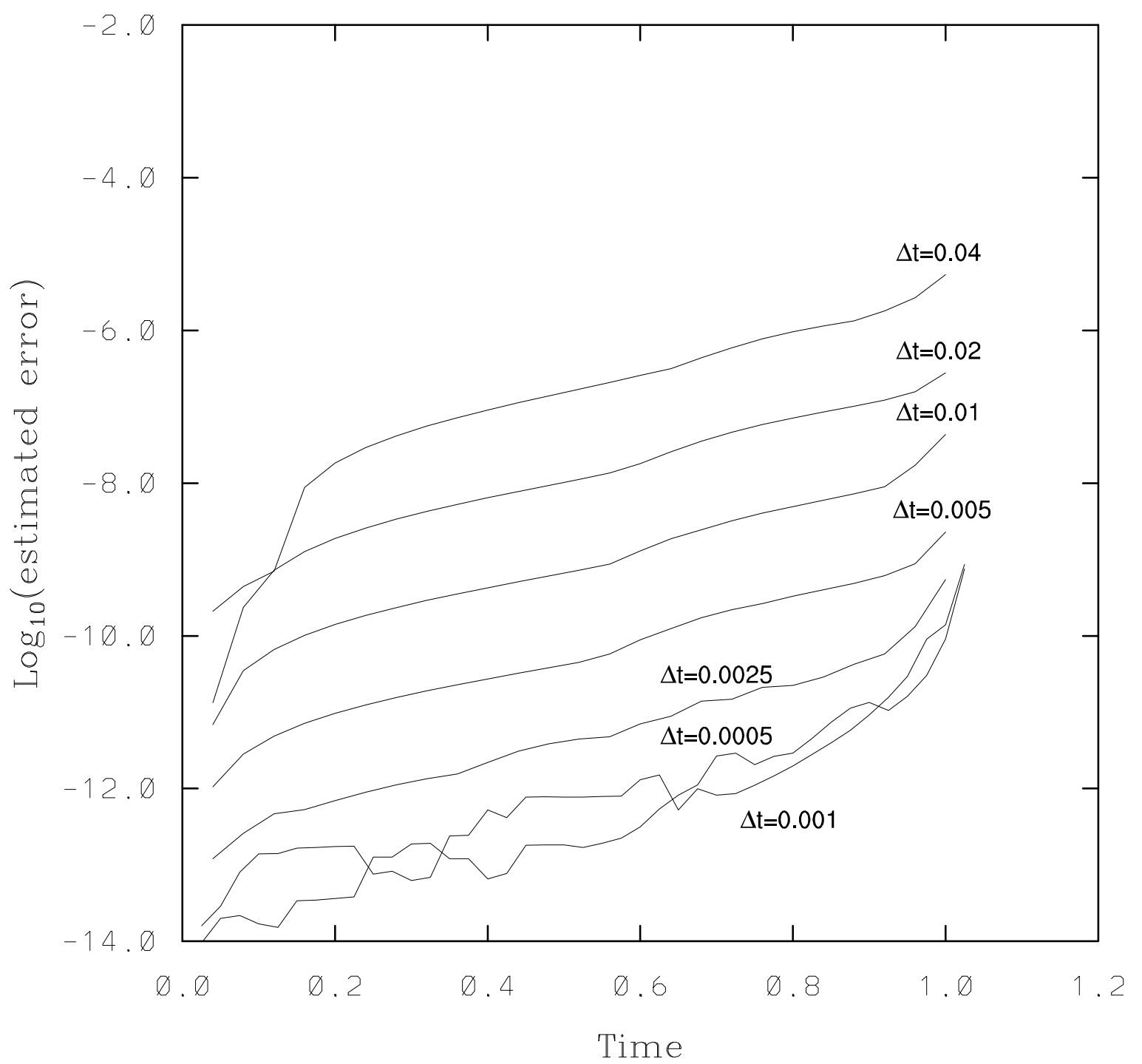

Figure 7: 


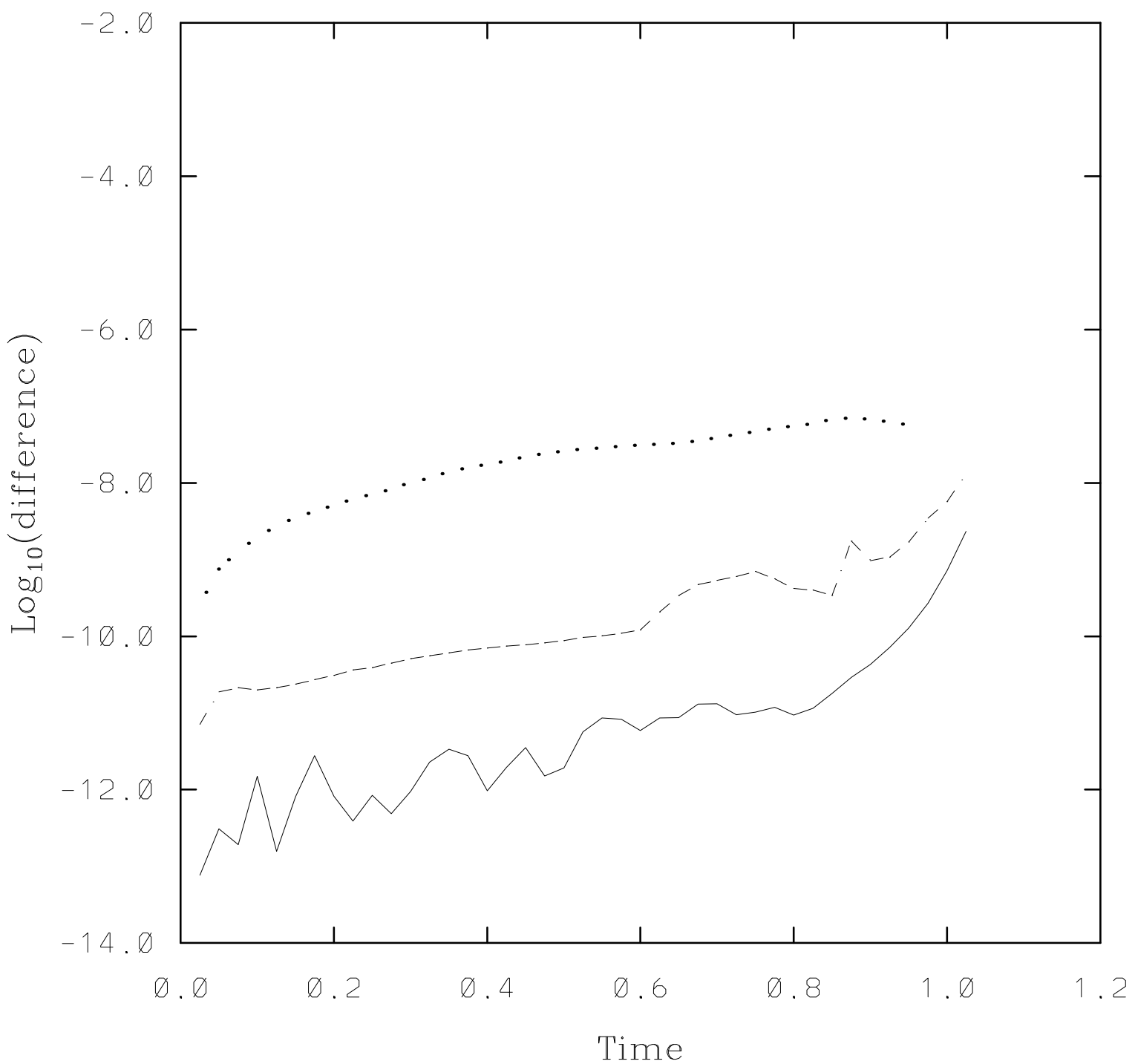

Figure 8: 


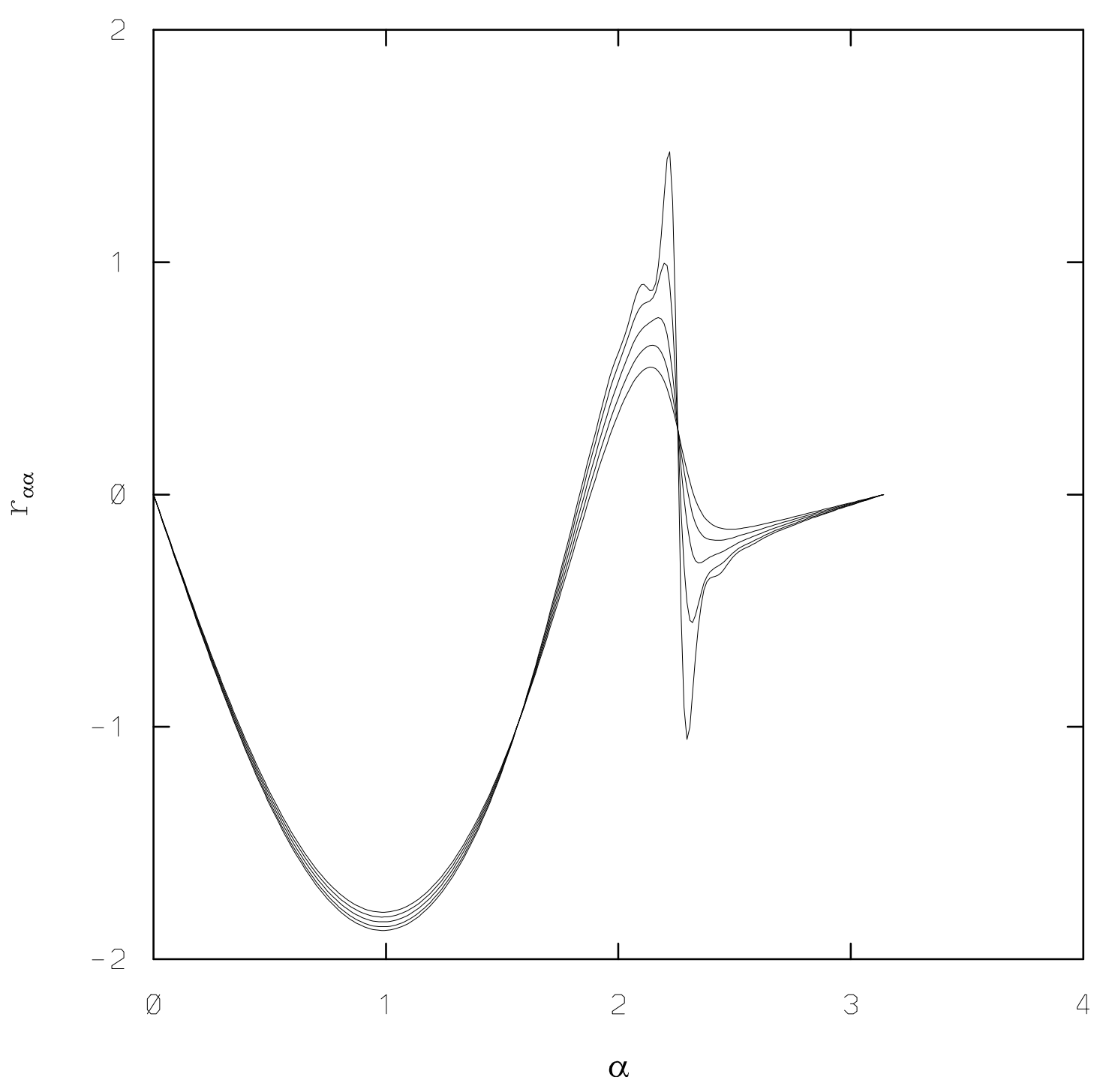

Figure 9: 